\title{
Real-time Automatic 3D Scene Generation from Natural Language Voice and Text Descriptions
}

\author{
Lee M. Seversky \\ Lee.Seversky@binghamton.edu \\ Lijun Yin \\ Lijun@cs.binghamton.edu \\ Department of Computer Science \\ State University of New York at Binghamton \\ PO Box 6000, Binghamton, NY 13902
}

\begin{abstract}
Automatic scene generation using voice and text offers a unique multimedia approach to classic storytelling and human computer interaction with $3 \mathrm{D}$ graphics. In this paper, we present a newly developed system that generates $3 \mathrm{D}$ scenes from voice and text natural language input. Our system is intended to benefit non-graphics domain users and applications by providing advanced scene production through an automatic system. Scene descriptions are constructed in real-time using a method for depicting spatial relationships between and among different objects. Only the polygon representations of the objects are required for object placement. In addition, our system is robust. The system supports different quality polygon models such as those widely available on the Internet.
\end{abstract}

\section{Categories and Subject Descriptors}

I.3.6 [Computer Graphics]: Methodology and TechniquesInteraction Techniques

\section{General Terms}

Algorithms, Human Factors

\section{Keywords}

Text-to-scene, Storytelling, 3D graphics, Natural language, Voice recognition, Real-time

\section{INTRODUCTION}

Manual scene composition in 3D is a time-consuming and intensive process. Interacting with $3 \mathrm{D}$ graphics-based media and applications requires users to adopt and learn specific graphics tools and interfaces. Such requirements limit the number of potential users and their interactions with a given media. Automatic scene generation reduces these requirements and makes 3D graphics more accessible to users in non-graphics domains. Using natural language descriptions in conjunction with automatic scene generation enables nongraphics areas to take advantage of the benefits of visualization in $3 \mathrm{D}$ while reducing the need for graphics specific knowledge. Additionally, real-time scene generation is ideal for collaborative and interactive applications. We present

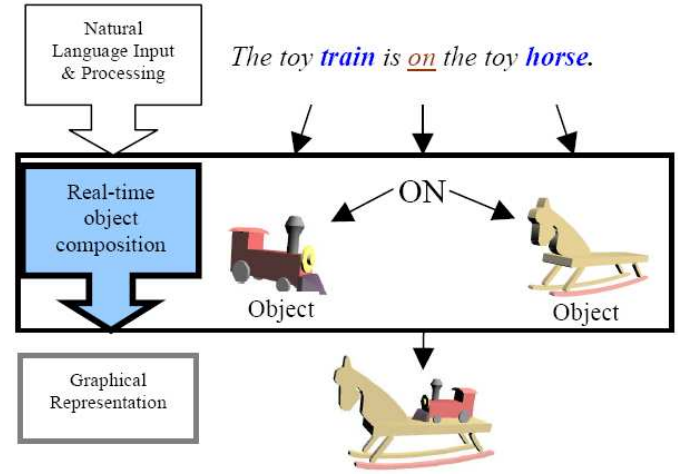

Figure 1: Real-time automatic scene composition framework.

a new system for automatically generating 3D scenes from voice and text descriptions in real-time. Figure 1 shows the components of our real-time composition system.

This work has significant impacts in any domain that can benefit from real-time 3D graphics and visualization such as education by using 3D scenes to reinforce concepts and ideas during a lesson, discussion, or story. Communication during collaborative environments can be improved by providing a central 3D scene that all members can interact with and see. Other areas such as law enforcement and the movie industry can also benefit from this work.

The presented system constructs a bridge between natural language input and graphical representation. The graphic depiction of a spatial relationship is dependent on the objects and their geometric representation. We focus on a real-time system for correctly depicting objects based on a given relationship. Figure 2 shows a generated scene using our system. The manual definition of per object depiction information is not required with our placement method.

The paper is divided into sections as follows: Section 2 gives an overview of similar composition systems. Section 3 describes in detail the placement algorithm used for scene composition. Section 4 describes our real-time scene composition system. Section 5 discusses our experiments. Section 6 discusses future work and in section 7 concluding remarks are presented. 
